\title{
Electrodynamics of Helium with Retardation and Self-Interaction Effects
}

\author{
Jayme De Luca \\ Instituto de Química de Araraquara-UNESP, Caixa Postal 355, Araraquara, São Paulo, 14801-970, Brazil
}

(Received 25 June 1997)

\begin{abstract}
We show that an extra constant of motion with an analytic form can exist in the neighborhood of some discrete circular orbits of helium when one includes retardation and self-interaction effects. The energies of these discrete stable circular orbits are in the correct atomic magnitude. The highest frequency in the stable manifold of one such orbit agrees with the highest frequency sharp line of parahelium to within $2 \%$. The generic term of the frequency in the stable manifold to higher orbits is also in agreement with the asymptotic form of quantum mechanics for helium. [S0031-9007(97)05078-3]
\end{abstract}

PACS numbers: 31.15.Ct, 03.20.+i, 05.45.+b

In this Letter we explore some surprising consequences of the retardation effects of Maxwell's electrodynamics to atomic physics. We show that electrodynamics with retardation prescribes a discrete set of stable circular orbits for the helium atom. The name "circular orbit" refers here to an orbit where the two electrons are performing the same circular motion and in phase opposition, that is, along a diameter [1].

The linearized dynamics about a circular orbit has a one-dimensional stable direction, a one-dimensional unstable direction, and ten neutrally stable directions [1]. For an infinitely massive $\alpha$ particle, helium has a sixdegree-of-freedom Hamiltonian system with only four independent constants of motion (namely, the energy and the three components of the total angular momentum). In the neighborhood of circular orbits, even if one restricts to the center manifold [2], one still has a five-degree-offreedom system. Because there are only four constants, the dynamics in the neighborhood of a generic circular orbit can be unstable, and actually is unstable in this case [1]. Here we show that an extra complex constant can exist in the neighborhood of a discrete set of circular orbits, when one includes retardation effects.

To include the retardation, we use the work of Page (1918) [3] on the expansion of the Liénard-Wiechert formula [4]. This formula is complicated because of the retardation constraint, and one way to convert it into a useful differential equation is to develop the constraint in a Taylor series [3]. Truncated to second order in $(v / c)$, this retarded interaction is still Lagrangian and is described by the Darwin Lagrangian [4]. The Darwin Lagrangian is rotationally invariant, which generates an angular-momentum-like constant according to Noether's theorem [5]. This constant is the mechanical angular momentum plus the small correction for the angular momentum of the electromagnetic field. There is also an energylike constant (because the Darwin Lagrangian is time independent).

According to standard normal form theory, from a resonance one can produce an extra complex constant about a fixed point [6]. Differently from the purely Coulombian case, in the presence of retardation the frequencies of the variational dynamics depend nonlinearly on the orbit's frequency. Because of this, an extra constant is possible for some discrete orbits, which is a genuine signature of the dynamics with retardation. We stress that it is absolutely essential to include the retardation to unfold a degeneracy of the Coulomb dynamics, and not to attain some better precision. Last, we consider only the dynamics of spinless pointlike charges, and issues like quantum mechanical spin are not discussed.

In this Letter we assume that the self-interaction effects are described by the Lorentz-Dirac equation (LDE) with a renormalized mass [4,7]. The LDE equation for an electron of charge $-e$ can be written in the convenient, noncovariant form as

$$
\frac{d}{d t}\left(\gamma M_{e} \dot{\mathbf{x}}_{e}\right)=\mathbf{F}_{\mathrm{rad}}+\mathbf{F}_{\mathrm{ext}},
$$

where $\dot{\mathbf{x}}_{e}$ is the electron's velocity, $M_{e}$ the renormalized electronic mass [7], and $\gamma \equiv 1 / \sqrt{1-\left(\left|\dot{\mathbf{x}}_{e}\right| / c\right)^{2}}$. In (1), $\mathbf{F}_{\text {ext }}$ is the external force, and in this Letter we use $\mathbf{F}_{\text {rad }}=\frac{2 e^{2}}{3 c^{3}} \dddot{\mathbf{x}}_{e}$, which is the lowest order approximation in powers of $(v / c)$ to the exact relativistic radiation force [7]. For a circular orbit, this is a term of order $(v / c)^{3}$.

We now introduce the expansion of the retardation constraint of the Liénard-Wiechert interaction, henceforth called the Page series [3]. Let $\mathbf{x}_{1}$ be the position of electron 1 , of charge $-e$, and $\beta_{1}$ its velocity vector divided by $c$. The formula for the electric field made by electron 1 at a position $\mathbf{x}_{2}$ is

$$
\mathbf{E}_{12}=-e \frac{\hat{\mathbf{n}}}{R_{12}^{2}}+\mathbf{E}_{12}^{(2)}\left(\mathbf{x}_{2}, \mathbf{x}_{1}, \dot{\mathbf{x}}_{1}, \ddot{\mathbf{x}}_{1}\right)+\mathbf{E}_{12}^{(3)}\left(\dddot{\mathbf{x}}_{1}\right)+\ldots,
$$

where

$$
\begin{gathered}
\mathbf{E}_{12}^{(2)} \equiv-e\left\{\frac{\left(\left|\beta_{1}\right|^{2}-3\left(\hat{\mathbf{n}} \cdot \beta_{1}\right)^{2}\right) \hat{\mathbf{n}}}{2 R_{12}^{2}}-\frac{\dot{\beta}_{1}}{2 c R_{12}}\right. \\
\left.-\frac{\left(\hat{\mathbf{n}} \cdot \dot{\beta}_{1}\right) \hat{\mathbf{n}}}{2 c R_{12}}\right\},
\end{gathered}
$$


and

$$
\mathbf{E}_{12}^{(3)}\left(\dddot{\mathbf{x}}_{1}\right) \equiv-\frac{2 e}{3 c^{3}} \dddot{\mathbf{x}}_{1},
$$

where $\hat{\mathbf{n}}$ is the unit vector in the direction of $\mathbf{x}_{2}-\mathbf{x}_{1}$ and $R_{12}=\left|\mathbf{x}_{2}-\mathbf{x}_{1}\right|$. Notice that all functions are evaluated at the present time. For a circular orbit, in units of the zeroth order Coulomb term, (3) is of order $(v / c)^{2}$ and (4) is of order $(v / c)^{3}$. We assume that the Taylor series of (2) is convergent or at least asymptotic for circular orbits, since $(v / c) \ll 1$ in atomic physics. The first term of the Page series for the magnetic field of electron 1 at $\mathbf{x}_{2}$ is [3]

$$
\mathbf{B}_{12}^{(1)}\left(\mathbf{x}_{2}, \mathbf{x}_{1}, \dot{\mathbf{x}}_{1}\right)=-\frac{e}{R_{12}^{2}}\left[\beta_{1} \times \hat{\mathbf{n}}\right] .
$$

In this Letter we consider only the above terms of the electromagnetic interaction, as well as the relativistic correction to the electronic dynamics up to order $(v / c)^{2}$.

Along a circular orbit, the total force acting on electron 2 can be calculated, using (3), (4), (5), and the selfinteraction of electron 2 , to be

$$
\mathbf{F}_{2}=\frac{2 e^{2}}{3 c^{3}}\left(\dddot{\mathbf{x}}_{1}+\dddot{\mathbf{x}}_{2}\right)-7 \frac{e^{2} \hat{\mathbf{n}}}{4 R^{2}}\left(1-\frac{3}{7}|\beta|^{2}\right),
$$

where $R$ is the radius of the circular orbit. In (6) we have also added the Coulomb attraction of the $\alpha$ particle (of charge $2 e$ ) that we suppose is infinitely massive and resting at the origin. Along the circular orbit, because the electrons are in phase opposition, the force along the velocity cancels out, demonstrating that the circular orbit is a possible periodic solution of the electromagnetic equations up to third order in $(v / c)$.

Notice the appearance of the dipole term in (6),

$$
\ddot{\mathbf{D}}=-e\left(\ddot{\mathbf{x}}_{1}+\ddot{\mathbf{x}}_{2}\right) .
$$

The total far field caused by the electrons depends linearly on the quantity $\ddot{\mathbf{D}}$ of (7), up to quadrupole terms [4]. If D is zero, the orbit does not radiate in dipole. The fifth-order quadrupole terms would be important only in a much longer time scale, of order $T /(v / c)^{5}$ [8]. In this Letter we consider only the dynamical system defined by truncating the Page series and self-interaction to third order in $(v / c)$.

Next we consider the dynamics of helium in the neighborhood of a circular orbit, according to the Darwin Lagrangian. For the Coulomb dynamics, this stability problem has already been considered by many authors $[1,9]$. Here we consider only the second order of the Page series, which is essential to unfold a degeneracy of the Coulomb dynamics. The detailed consideration of the dissipative effect of the third-order term of the Page series and the (also third-order) self-interaction term will be published elsewhere [8]. Here it suffices to say that these higher-order terms are important only in a much longer time scale. To second order in $(v / c)$, the equations of motion for helium with retardation and relativistic correction are

$$
\begin{aligned}
M_{e} \frac{d}{d t}\left[\left(1+\frac{1}{2}\left|\dot{\mathbf{x}}_{1}\right|^{2}\right) \dot{\mathbf{x}}_{1}\right]= & -\frac{2 e^{2}}{R_{1}^{3}}\left(\mathbf{x}_{1}\right)-\frac{e^{2}}{R_{12}^{3}}\left(\mathbf{x}_{2}-\mathbf{x}_{1}\right) \\
& +\mathbf{E}_{21}^{(2)}\left(\mathbf{x}_{1}, \mathbf{x}_{2}, \dot{\mathbf{x}}_{2}, \ddot{\mathbf{x}}_{2}\right) \\
& -\frac{e}{c} \dot{\mathbf{x}}_{1} \times \mathbf{B}_{21}^{(1)}\left(\mathbf{x}_{1}, \mathbf{x}_{2}, \dot{\mathbf{x}}_{2}\right), \\
M_{e} \frac{d}{d t}\left[\left(1+\frac{1}{2}\left|\dot{\mathbf{x}}_{2}\right|^{2}\right) \dot{\mathbf{x}}_{2}\right]= & -\frac{2 e^{2}}{R_{2}^{3}}\left(\mathbf{x}_{2}\right)-\frac{e^{2}}{R_{12}^{3}}\left(\mathbf{x}_{1}-\mathbf{x}_{2}\right) \\
& +\mathbf{E}_{12}^{(2)}\left(\mathbf{x}_{2}, \mathbf{x}_{1}, \dot{\mathbf{x}}_{1}, \ddot{\mathbf{x}}_{1}\right) \\
& -\frac{e}{c} \dot{\mathbf{x}}_{2} \times \mathbf{B}_{12}^{(1)}\left(\mathbf{x}_{2}, \mathbf{x}_{1}, \dot{\mathbf{x}}_{1}\right),
\end{aligned}
$$

where $M_{e}$ is the electronic mass, $R_{1}=\left|\mathbf{x}_{1}\right|, R_{2}=$ $\left|\mathbf{x}_{2}\right|, R_{12}=\left|\mathbf{x}_{1}-\mathbf{x}_{2}\right|, \mathbf{E}_{12}^{(2)}\left(\mathbf{x}_{2}, \mathbf{x}_{1}, \dot{\mathbf{x}}_{1}, \ddot{\mathbf{x}}_{1}\right)$ is defined in (3), and $\mathbf{B}_{12}^{(1)}\left(\mathbf{x}_{2}, \mathbf{x}_{1}, \dot{\mathbf{x}}_{1}\right)$ is defined in (5). The circular orbit is the following periodic solution of (8):

$$
\begin{array}{cl}
x_{1}=R \cos (\omega t), & y_{1}=R \sin (\omega t), \quad z_{1}=0, \\
x_{2}=-R \cos (\omega t), & y_{2}=-R \sin (\omega t), \quad z_{2}=0 .
\end{array}
$$

According to (8), the frequency of the orbit is related to $R$ by

$$
M_{e} \omega^{2}=\frac{7 e^{2}}{4 R^{3}}\left(1-\frac{3}{7}|\beta|^{2}\right),
$$

which is correct to second order in $(v / c)$.

Linearizing (8) about the circular orbit (9), we obtain a parametric linear differential equation with coefficients periodic in time and period $T=\pi / \omega$ [8]. If the Floquet exponents are all nondegenerate, one can find a complete set of solutions of the form [10]

$$
\begin{aligned}
& \delta \mathbf{x}_{1}=\exp (2 i \omega \mu t) \sum_{n} \mathbf{x}_{n}^{1} \exp (2 i n \omega t), \\
& \delta \mathbf{x}_{2}=\exp (2 i \omega \mu t) \sum_{n} \mathbf{x}_{n}^{2} \exp (2 i n \omega t),
\end{aligned}
$$

where the Floquet exponent $\mu$ is a complex number defined in the first Brillouin zone, $-1 / 2<\operatorname{Re}(\mu)<$ $1 / 2$. Notice that for the Floquet components we use an upper index, but to label coordinates as functions of time, as in (8), we use a lower index (to distinguish it from the Floquet components). To simplify the variational equations, we define the coordinates

$$
\xi_{n}^{\kappa} \equiv \frac{1}{\sqrt{2}}\left(x_{n}^{\kappa}-i y_{n}^{\kappa}\right), \quad \chi_{n}^{\kappa} \equiv \frac{1}{i \sqrt{2}}\left(x_{n}^{\kappa}+i y_{n}^{\kappa}\right) .
$$

Next we calculate Hill's secular determinant [10] for the linearization of (8) about (9). As a simplification, let us define $\bar{n} \equiv n+\mu$ to be the running variable in the summations of (11). Last, to introduce physical intuition, 
it is convenient to define the radiation coordinate $\mathbf{x}_{r}$ and relative coordinate $\mathbf{x}_{d}$ as

$$
\begin{aligned}
& \mathbf{x}_{r} \equiv \mathbf{x}_{1}+\mathbf{x}_{2}, \\
& \mathbf{x}_{d} \equiv \mathbf{x}_{1}-\mathbf{x}_{2} .
\end{aligned}
$$

It turns out, as one can easily verify, that the linearized dynamics for the $\delta \mathbf{x}_{d}$ variable is decoupled from the $\delta \mathbf{x}_{r}$ variation. Here, for lack of space, we only develop the dynamics of the $\delta \mathbf{x}_{d}$ variable along the $z=0$ plane, correct to second order in $(v / c)$. We refer to [1] for the Coulomb eigenvalues of the $\delta \mathbf{x}_{r}$ dynamics. A complete study of the eigenvalues will be published elsewhere [8]. Before we write the planar variational equations, we need still another definition:

$$
\begin{aligned}
U_{n}^{\kappa} & \equiv \frac{1}{\sqrt{2}} \xi_{n+1}^{\kappa}+\frac{i}{\sqrt{2}} \chi_{n-1}^{\kappa}, \\
V_{n}^{\kappa} & \equiv \frac{-i}{\sqrt{2}} \xi_{n+1}^{\kappa}-\frac{1}{\sqrt{2}} \chi_{n-1}^{\kappa} .
\end{aligned}
$$

We make the quantities $U_{n}^{\kappa}$ and $V_{n}^{\kappa}$ into the $x$ and $y$ components of a bidimensional vector $\mathbf{K}_{n}^{\kappa} \equiv\left(U_{n}^{\kappa}, V_{n}^{\kappa}\right)$, and also define $\tilde{\mathbf{K}}_{n}^{\kappa} \equiv\left(-V_{n}^{\kappa}, U_{n}^{\kappa}\right), \mathbf{x}_{n} \equiv\left(x_{n}, y_{n}\right)$, and $\tilde{\mathbf{x}}_{n} \equiv$ $\left(-y_{n}^{\kappa}, x_{n}^{\kappa}\right)$. The variational equations for planar motion along the variable $\delta \mathbf{x}_{d}$ can be written most simply in Floquet components as [8]

$$
\begin{gathered}
-4 \bar{n}^{2} \mathbf{x}_{n}^{d}-\frac{1}{2}\left(\mathbf{x}_{n}^{d}+3 \mathbf{K}_{n}^{d}\right)+ \\
|\beta|^{2}\left(P(\bar{n}) \mathbf{x}_{n}^{d}+Q(\bar{n}) \mathbf{K}_{n}^{d}-\frac{6 i \bar{n}}{7} \tilde{\mathbf{x}}_{n}^{d}+\frac{18 i \bar{n}}{7} \tilde{\mathbf{K}}_{n}^{d}\right)=0 .
\end{gathered}
$$

We recall that $\bar{n}=n+\mu$, and there is one equation for every value of $n$. In principle, this would lead to an infinite Hill's secular determinant [10]. For the case of circular orbits, when we use the $\xi$ and $\chi$ variables, we find that the $\xi_{n}$ variables couple only to $\chi_{n+1}$ and vice versa. The variational problem for the coordinate $\delta \mathbf{x}_{d}$, as defined by (16), turns into the following $2 \times 2$ matrix equation

$$
\begin{aligned}
& {\left[\begin{array}{cc}
-\left(\frac{1}{2}+4 \bar{n}^{2}\right) & \frac{-3 i}{2} \\
\frac{3 i}{2} & -\left(\frac{1}{2}+4 \bar{n}_{+}^{2}\right)
\end{array}\right]\left[\begin{array}{c}
\xi_{n}^{d} \\
\chi_{n+1}^{d}
\end{array}\right]+} \\
& |\beta|^{2}\left[\begin{array}{cc}
P_{2}(\bar{n}) & i Q_{2}(\bar{n}) \\
-i Q_{2}\left(-\bar{n}_{+}\right) & P_{2}\left(-\bar{n}_{+}\right)
\end{array}\right]\left[\begin{array}{c}
\xi_{n}^{d} \\
\chi_{n+1}^{d}
\end{array}\right]=0,
\end{aligned}
$$

where $\quad P_{2}(\bar{n}) \equiv\left(-\frac{4 \bar{n}^{2}}{7}+\frac{6 \bar{n}}{7}+\frac{3}{28}\right), Q_{2}(\bar{n}) \equiv\left(\frac{18 \bar{n}^{2}}{7}-\right.$ $\left.\frac{18 \bar{n}}{7}+\frac{1}{28}\right)$, and $\bar{n}_{+} \equiv(1+\bar{n})$. We now consider the secular equation associated with Eq. (17): At $|\beta|=0$, we obtain the eigenvalues of the Coulomb stability problem,

$$
\bar{n}=0,-1,-1 / 2,-1 / 2 .
$$

The next step is to consider the secular equation of (17) for finite values of $|\beta|$. The essential ingredient for us here is the degenerate root $\bar{n}=-1 / 2$ of (18). Because of this degeneracy, the double root $\bar{n}=-1 / 2$ acquires a correction proportional to $|\beta|$, instead of the generic second-order correction of the other nondegenerate roots. Solving the secular equation of (17), in the neighborhood of $\bar{n}=-1 / 2$ and in the lowest order approximation in $|\beta|$, we find

$$
\bar{n} \simeq-1 / 2 \pm \sqrt{\frac{51}{28}}|\beta| .
$$

According to (11), $\bar{n}$ is a frequency of oscillation in units of $2 \omega$. For example, along the linear mode of (17) defined by $\bar{n}=-1 / 2$, the frequencies in (11) are $-\omega=2 \omega \bar{n}$ and $\omega=2 \omega(1+\bar{n})$.

The circular orbit is a fixed point of the autonomous vector field describing the dynamics in the system rotating with the frequency of the circular orbit. To apply resonant normal form theory $[6,8]$, we must move to this rotating system and find a new nontrivial resonance. In this rotating system, the new frequency for oscillation along the mode (19) is found by adding $1 / 2$ to (19). In units of $2 \omega$, the frequency of Coulombic oscillations along the $z$ direction in the mode $\delta z_{r}$ is found in [1] to be

$$
\omega_{1} \equiv \sqrt{\frac{2}{7}} \simeq 0.5345 \ldots
$$

and for planar Coulombic oscillations along the mode $\delta \mathbf{x}_{r}$, in the rotating frame, is [1]

$$
\omega_{2} \equiv \sqrt{\frac{3+\sqrt{32}}{28}} \simeq 0.5560 \ldots
$$

also in units of $2 \omega$. A new resonance condition involving the root (19) is the easiest to be satisfied for small values of $|\beta|$. therefore, we are looking for a resonance among the frequencies of (21), (20), and (19), which in the rotating frame evaluates to

$$
\omega_{3} \simeq \sqrt{\frac{51}{28}}|\beta| \simeq 1.3496|\beta|,
$$

in units of $2 \omega$. By inspection, we find that the resonance involving the above three frequencies and with the minimal integer multipliers is of type

$$
\omega_{1}-\omega_{2}+2 n \omega_{3}=0,
$$

where $n$ is an integer number and the factor of 2 multiplying $\omega_{3}$ is because mode 3 comes from a degenerate eigenvalue. The reason for the special combination of (23) should be sought in the normal form and will be discussed elsewhere [8]. Condition (23) is satisfied for $|\beta|$ given by

$$
|\beta| \simeq \frac{0.00797}{n} .
$$

The binding energies, in atomic units, for the above values of $|\beta|$ are

$$
E=-\frac{2.3853}{n^{2}},
$$


and the frequencies of the special "stable" circular orbits described by (23), in atomic units, are given by

$$
\omega=\frac{0.7442}{n^{3}} \text {. }
$$

Because we are involving a resonance among the planar oscillations $\delta \mathbf{x}_{r}, \delta \mathbf{x}_{d}$, and the $\delta z_{r}$ oscillation, the leading term of the normal form is a product of $\delta z_{r}$ times the planar coordinate variations. In reference [8] we show how to construct this extra constant and discuss the shape of its level surfaces.

The frequency $\omega_{z}$ for oscillations along the $\delta z_{r}$ linear mode can be obtained, as prescribed by (20), by multiplying the above frequency by $2 \sqrt{2 / 7}$,

$$
\omega_{z}=\frac{0.7956}{n^{3}} \text {. }
$$

The inclusion of the third-order radiative terms makes this linear model of (27) a stable mode [8], whereby the perturbation radiates energy and decays to the perfectly circular orbit. The frequency of this stable oscillation should then appear in the spectrum of sharp lines, given that the dynamics selects the neighborhood of the stable orbits. For example, the transition $2^{1} P \rightarrow 1^{1} S$ of parahelium corresponds to a frequency of $2.9037-$ $2.1237=0.7799$ atomic units [11,12], and (27) with $n=$ 1 evaluates to 0.7956 , which is a $2 \%$ difference. The transition $3^{1} P \rightarrow 2^{1} S$ in parahelium [12] has a frequency of $2.1459-2.0551=0.0908$ atomic units, and (27) with $n=2$ evaluates to 0.0995 , which is an $8 \%$ difference. The asymptotic form of the quantum mechanical energies of helium are [11]

$$
E=-\frac{Z^{2}}{2}-\frac{(Z-1)^{2}}{2\left(n_{r}+L+1\right)^{2}},
$$

with $Z=2$. For transitions with $\Delta L=1$, the frequencies predicted from the above formula are given by

$$
\omega_{q}=\frac{1}{\left(n_{r}+L+1\right)^{3}},
$$

which agrees with (27) to within $20 \%$. We stress that we relied only on dynamics to calculate the frequency of (27). Equation (29), on the other hand, was obtained in the usual way of taking differences between quantum energies, and was included only for comparison.

A more detailed comparison with the spectrum of helium requires the consideration of elliptical orbits and zero-angular-momentum orbits [8]. In Ref. [8] we include the third-order terms and find that the resulting damping of the oscillation mode of (27) agrees well with the quantum calculations of the linewidth.
The extra complex constant is actually two real functions of phase space [8]. Together with the four (three in involution) global constants of helium, this can produce a barrier in phase space, at least inside a small resonance region. This should stabilize the dynamics in a short time scale, possibly by creating a simple homoclinic return for the unstable direction (radial instability [1,9]). Notice that to emit a sharp line only finite time stability is needed, so resonant normal form is the perfect tool here.

Historically, the radial instability [1], first found by Nicholson [9], led Bohr to postulate that, along a quantized set of orbits, some special mechanism would supersede the Coulombian radial instability, thus stabilizing the atom, which proved to be a very fruitful intuition [13]. Some of the details of how the extra complex constant stabilizes the dynamics, in a time scale of order $T /(v / c)^{2}$, are discussed elsewhere [8].

For a short review of applications of Eq. (1) to atomic hydrogen, one should see Ref. [14].

The existence of this extra constant is a genuine effect of electrodynamics with retardation. It is surprising that classical electrodynamics with retardation can offer such a sensible approximation to the spectrum of sharp lines. We do not know of any prior similar application of dynamics to atomic physics.

I acknowledge discussions with R. Napolitano and J. C. Egues. This work was supported by Fapesp.

[1] M. Poirier, Phys. Rev. A 40, 3498 (1989).

[2] J. Guckenheimer and P. Holmes, Nonlinear Oscillations, Dynamical Systems and Bifurcations of Vector Fields (Springer, New York, 1983).

[3] L. Page, Phys. Rev. 11, 376 (1918).

[4] J.D. Jackson, Classical Electrodynamics (Wiley, New York, 1975).

[5] V.I. Arnold, Mathematical Methods of Classical Mechanics (Springer-Verlag, New York, 1978).

[6] F. Gustavson, Astron. J. 71, 670 (1966).

[7] S. Coleman, in Electromagnetism, Paths to Research, edited by D. Teplitz (Plenum, New York, 1982).

[8] J. De Luca (to be published).

[9] J. W. Nicholson, Mon. Not. R. Astron. Soc. LXXII, 49 (1912); LXXII, 139 (1912); LXXII, 677 (1912); LXXII, 693 (1912); LXXII, 729 (1912).

[10] D. W. Jordan and P. Smith, Nonlinear Ordinary Differential Equations (Clarendon, Oxford, 1977).

[11] H. A. Bethe and E.E. Salpeter, Quantum Mechanics of One- and Two-electron Atoms (Plenum, New York, 1977).

[12] Ming-Keh Chen, J. Phys. B 27, 4847 (1994).

[13] N. Bohr, Philos. Mag. 26, 1 (1913); 26, 476 (1913).

[14] J. De Luca, Braz. J. Phys. 27, 285 (1997). 